Claremont Colleges

Scholarship@ Claremont

All HMC Faculty Publications and Research

HMC Faculty Scholarship

2-1-1993

\title{
A Theoretical Investigation of the Geometries, Vibrational Frequencies, and Binding Energies of Several Alkali Halide Dimers
}

\author{
Robert P. Dickey'93 \\ Harvey Mudd College \\ David Maurice '91 \\ Harvey Mudd College \\ Robert J. Cave \\ Harvey Mudd College \\ Richard J. Mawhorter \\ Pomona College
}

\section{Recommended Citation}

A theoretical investigation of the geometries, vibrational frequencies, and binding energies of several alkali halide dimers. Robert P. Dickey, David Maurice, Robert J. Cave, and Richard Mawhorter, J. Chem. Phys. 98, 2182 (1993), DOI: 10.1063/1.464197

This Article is brought to you for free and open access by the HMC Faculty Scholarship at Scholarship @ Claremont. It has been accepted for inclusion in All HMC Faculty Publications and Research by an authorized administrator of Scholarship @ Claremont. For more information, please contact scholarship@cuc.claremont.edu. 


\title{
A theoretical investigation of the geometries, vibrational frequencies, and binding energies of several alkali halide dimers
}

\author{
Robert P. Dickey, David Maurice, ${ }^{\text {a) }}$ and Robert J. Cave ${ }^{\text {b) }}$ \\ Department of Chemistry, Harvey Mudd College, Claremont, California 91711 \\ Richard Mawhorter ${ }^{\text {b) }}$ \\ Department of Physics, Pomona College, Claremont, California 91711
}

(Received 10 July 1992; accepted 14 October 1992)

\begin{abstract}
Results are presented from $a b$ initio calculations on the symmetrical alkali halide dimers made up of $\mathrm{Li}, \mathrm{Na}, \mathrm{K}, \mathrm{F}$, and $\mathrm{Cl}$. We examine the sensitivity of representative monomer and dimer geometries to the variation of the basis set with and without polarization and diffuse functions. The geometries are then compared with available experimental results. We have also calculated vibrational frequencies at the restricted Hartree-Fock level and examined the changes in geometry brought about by correlation using second-order Møller-Plesset perturbation theory. It is found that Hartree-Fock theory in a modest basis set with diffuse and polarization functions yields results comparable to much larger $s p$ basis sets and that the theoretical results are in good agreement with the experimental results for the $\mathrm{Li}$ and $\mathrm{Na}$ dimers. Our best results for the $\mathrm{K}$-containing species tend to have bond lengths that are too long for the monomers and this error is carried over for the dimers. We also find a nearly uniform expansion of the $\mathrm{M}-\mathrm{X}$ bond length in proceeding from monomer to dimer of $0.16 \pm 0.03 \AA$, independent of the alkali or halide involved. The calculated dimer dissociation energies are in excellent agreement with experiment. Inclusion of correlation appears to have a minimal effect on the computed geometries and a modest effect on the binding energies. The vibrational frequencies for the monomers are in excellent agreement with experimental gas-phase results and reasonable agreement is obtained with the available experimental frequencies for the dimers. Finally, a reanalysis of the electron diffraction data for $\mathrm{Na}_{2} \mathrm{~F}_{2}$ in light of the current $a b$ initio results leads to a significant change in the experimental value of the bond angle.
\end{abstract}

\section{INTRODUCTION}

The geometry of alkali halide crystals is a common subject in undergraduate chemistry courses due to the relative simplicity of the bonding in the crystals and the simple crystal structures they exhibit. The monomers are also particularly simple and their bonding can be represented semiquantitatively by ionic models with inclusion of repulsive corrections for the cores. ${ }^{1-8}$ The more difficult question comes when one considers intermediate size clusters of alkali halide molecules. ${ }^{9-14}$ The nature of the stable geometries, the energetic nearness of other local minima, and the point at which such clusters become crystalline-like are all questions of current interest. ${ }^{15,16} \mathrm{~A}$ recent series of electron diffraction studies on the geometries of the dimers of the alkali halides ${ }^{11-13}$ sparked us to consider several of the dimers from a theoretical viewpoint.

That vapors of alkali halides contain aggregated species has been known for nearly 40 years. ${ }^{17}$ Early attempts at characterizing the aggregates determined that the major nonmonomeric species were dimers, while gas-phase ${ }^{18}$ and matrix-isolation IR spectra ${ }^{17,19-21}$ were used to determine vibrational frequencies for the dimers. Several semiempirical theoretical methods were also used to estimate geom-

\footnotetext{
${ }^{a}$ Current address: Department of Chemistry, University of California at Berkeley, Berkeley, CA 94720.

b) Authors to whom correspondence should be addressed.
}

etries and vibrational frequencies with reasonable success. $^{1-8}$ In some cases, $a b$ initio geometries have been obtained as well, such as for $\mathrm{Li}_{2} \mathrm{~F}_{2}$ (Ref. 22) and $\mathrm{Na}_{2} \mathrm{Cl}_{2}{ }^{23,24}$ The more detailed structural data provided by electron-diffraction studies, however, poses some questions of previous theory. In particular, the various theories only yield modest agreement with experiment on the internal angles for the dimers, and quantitative agreement with dimer bond lengths was not achieved. Furthermore, the experiments seem to indicate that the $\mathrm{K}$-containing dimers have anomalously long $M-X$ bond lengths, and that the $\mathrm{Rb}-\mathrm{X}$ and $\mathrm{Cs}-\mathrm{X}$ bond lengths are in some cases identical, even though $\mathrm{Rb}^{+}$and $\mathrm{Cs}^{+}$have ionic radii that differ by on the order of $0.2 \AA^{25}$

The present study was aimed at examining several of the areas of disagreement between the more recent experiments and past theory for the symmetric dimers containing $\mathrm{Li}, \mathrm{Na}, \mathrm{K}, \mathrm{F}$, and $\mathrm{Cl}$. We examined the series of geometries at higher levels of theory than have been applied previously to most of the dimers and examined the effects of variations in the one-particle basis set. The effects of electron correlation were also studied. The trends that have been obtained indicate that the $\mathrm{K}$-containing dimers should behave similarly to the light alkali-containing dimers, and thus our results suggest further analysis is needed for $\mathrm{K}_{2} \mathrm{Cl}_{2}$. Finally, we obtained vibrational frequencies for the IR and Raman active modes of all six dimers. 
Reasonable agreement was obtained with the (limited) past experimental results.

The remainder of the paper is organized as follows: In Sec. II, we outline the theoretical methods used in the present study. In Sec. III, we present our results, and the results are discussed in Sec. IV. Our conclusions are presented in Sec. V.

\section{THEORETICAL METHODS}

The restricted Hartree-Fock (RHF) and restricted open-shell Hartree-Fock (ROHF) calculations, geometry optimizations, vibrational analyses, and second-order Møller-Plesset (MP2) calculations were performed using GAMESS. ${ }^{26}$ MELDF $^{27}$ was used to perform spherically averaged Hartree-Fock calculations in order to contract the largest basis sets for the halide atoms.

All reported dimer geometries have $D_{2 h}$ symmetry. Linear geometries were examined, but were found to be higher in energy than the $D_{2 h}$ structures and were unstable with respect to bending. Nonplanar $C_{2 v}$ geometries were not sought since the $D_{2 h}$ structures were found to be true minima in the vibrational analyses and since it was felt that bending out of plane tended to bring the two like-charged atoms closer, leading to greater electrostatic repulsion. The vibrational analyses were performed at the computed stationary points. The convergence to a stationary point has a numerical tolerance associated with it; these are only approximately "true" stationary points and we estimate that they are accurate to $\pm 0.001 \AA$. This leads to an associated uncertainty in the vibrational frequencies which we estimate to be no more than $5 \mathrm{~cm}^{-1}$. This uncertainty will have no effect on the conclusions we draw below.

A wide variety of basis sets were considered in the present study ranging from modest valence double zeta sets to contracted basis sets based on large even-tempered primitive spaces. It was found that the results of the largest basis sets were reproduced quite well by a 6-31G basis augmented with diffuse and polarization functions, which are outlined below. In the case of NaF and its dimer, however, we present results from a variety of basis sets to outline the features of importance in the calculations. When the standard diffuse functions or polarization functions are used, we follow the convention that basis set + implies the basis set augmented with diffuse functions, and that basis set $*$ indicates the basis set augmented with $d$ polarization functions.

Li. The Pople $6-31 \mathrm{G}^{28}$ set was augmented with one set of $d$ functions $(\alpha=0.2)$ and two sets of $p$ polarization functions $(\alpha=1.0$ and 0.33$)$. We denote this below as our "polarized basis" or "POL."

Na. The Pople $3-21 G^{29}$ and $6-31 G^{28}$ basis sets were used. In some cases, these sets were augmented with standard diffuse $s$ and $p$ functions $(\alpha=0.0076)$ and a set of $d$ polarization functions $(\alpha=0.175)$. In most cases, however, we used the 6-31G basis and augmented it with two sets of $d$ functions $(\alpha=1.0$ and 0.175$)$. This is denoted as the polarized basis below. We also used an even-tempered $(18 s, 8 p / 5 s, 3 p)$ basis $^{30}$ contracted using a Raeffenetti scheme $^{31}$ based on the atomic ROHF calculation. The first three $s$ functions were contractions based on the $1 s, 2 s$, and $3 s$ atomic orbitals of the first 16 basis functions and the final two $s$ functions were the two most diffuse $s$ functions. The first $p$ function was a contraction based on the atomic $2 p$ function and the last two $p$ functions were the uncontracted two most diffuse $p$ basis functions. This basis was augmented with $d$ polarization functions having exponents $\alpha=1.0$ and 0.175 . We designate this basis as "extended" or "EXT" below.

K. We used the McLean and Chandler $(14 s, 9 p)$ basis contracted to $(8 s, 4 p)^{32}$ augmented with two $d$ functions $(\alpha=0.5,0.1)$ and one $p$ function $(\alpha=0.5)$

F. The Pople $3-21 G^{29}$ and $6-31 G^{28}$ basis sets were used. In some cases, these sets were augmented with diffuse $s$ and $p$ functions $(\alpha=0.1076)$ and a set of $d$ polarization functions $(\alpha=0.8)$. When the 6-31G basis is used with these polarization and diffuse functions, it is denoted as the polarized basis; note that these are the same as the standard diffuse and polarization functions. We also used an even-tempered $(16 s, 8 p / 4 s, 3 p)$ basis $^{30}$ contracted using a Raeffenetti scheme ${ }^{31}$ based on the atomic spherically averaged ROHF orbitals ( $1 s, 2 s, 2 p$ functions plus the two most diffuse functions in each angular momentum). A $d$ polarization function was also used with exponent 0.8 and an extra diffuse $p$ function with exponent 0.0623 . This basis is denoted "EXT" below. In addition, we have also split the $F d$ function into a pair of $d$ functions $(\alpha=1.12$ and 0.32$)$ and this basis is denoted "EXT2" below.

Cl. The Pople $6-31 \mathrm{G}^{28}$ basis sets was used, augmented with diffuse $s$ and $p$ functions $(\alpha=0.0483)$ and one set of $d$ polarization functions $(\alpha=0.75)$. This basis is denoted as polarized below, but here again, the diffuse and polarization functions are the standard values. In test calculations (not reported), we have split the $\mathrm{Cl} d$ function used above into two functions (splitting factors ${ }^{26}$ of 0.4 and 1.4) and computed the geometries and vibrational frequencies of $\mathrm{LiCl}$ and $\mathrm{NaCl}$ and the geometries and dimerization energies of $\mathrm{Li}_{2} \mathrm{CL}_{2}$ and $\mathrm{Na}_{2} \mathrm{Cl}_{2}$. Negligible changes were found in the bond length expansions (at most $0.003 \AA$ ) and the dimer bond angles widened by about one degree. The absolute bond lengths changed by at most $0.01 \AA$ and the dimerization energies changed by at most $0.05 \mathrm{kcal} /$ mol. Because of the relative insensitivity to splitting the $d$ functions, we present results with the single set of $d$ functions below, and have only used the single $d$ function in calculations on $\mathrm{KCl}$ and $\mathrm{K}_{2} \mathrm{Cl}_{2}$.

\section{RESULTS}

In this section, theoretical results are presented for the energies and geometries of the alkali halide dimers. For clarity, the data are broken down into subsections concerning: (i) basis set quality and effects on geometries; (ii) geometries, vibrational frequencies, and binding energies at the self-consistent field (SCF) level; and (iii) correlation effects.

\section{A. Basis sets}

We have examined a variety of basis sets for the calculations of the monomer and dimer geometries and found 
TABLE I. Basis set comparison for NaF monomer and dimer geometries. $^{\mathrm{a}}$

\begin{tabular}{|c|c|c|c|c|}
\hline Mon/Dim & Basis set & $R_{\mathrm{MX}}$ & $\theta_{\mathrm{XMX}}$ & $\Delta R_{\mathrm{MX}}$ \\
\hline Mon & $3-21 G$ & 1.862 & & \\
\hline Mon & $3-21+G$ & .1 .903 & & \\
\hline Mon & $3-21 G^{*}$ & 1.813 & & \\
\hline Mon & $3-21+G^{*}$ & 1.874 & & \\
\hline Mon & 3-21G POL & 1.898 & & \\
\hline Mon & $6-31 G^{*}$ & 1.885 & & \\
\hline Mon & $6-31+G^{*}(+$ on $F)$ & 1.928 & & \\
\hline Mon & POL & 1.923 & & \\
\hline Mon & MP2 POL & 1.942 & & \\
\hline Mon & EXT & 1.923 & & \\
\hline Mon & EXT/EXT2 & 1.924 & & \\
\hline Mon & Experiment $^{\mathrm{b}}\left(R_{e}\right)$ & 1.926 & & \\
\hline Mon & Experiment $^{\mathrm{c}}\left(R_{e}\right)$ & $1.917(2)$ & & \\
\hline Mon & Experiment $^{\mathrm{d}}\left(\boldsymbol{R}_{a}\right)$ & $1.944(2)$ & & \\
\hline Mon & Experiment $t^{c}$ & 1.944 & & \\
\hline Dim & $3-21 G$ & 2.001 & 90.9 & 0.139 \\
\hline Dim & $3-21+G$ & 2.013 & 91.8 & 0.110 \\
\hline Dim & $3-21 G^{*}$ & 1.935 & 107.0 & 0.122 \\
\hline Dim & $3-21+G^{*}$ & 1.959 & 101.3 & 0.085 \\
\hline Dim & 3-21G POL & 2.008 & 94.1 & 0.110 \\
\hline Dim & $6-31 G^{*}$ & 2.040 & 94.0 & 0.155 \\
\hline Dim & $6-31+\mathrm{G}^{*}(+$ on $\mathrm{F})$ & 2.068 & 93.6 & 0.140 \\
\hline Dim & POL & 2.065 & 93.1 & 0.142 \\
\hline Dim & MP2 POL & 2.094 & 92.9 & 0.152 \\
\hline Dim & EXT & 2.065 & 93.1 & 0.142 \\
\hline Dim & EXT/EXT2 & 2.065 & 93.8 & 0.141 \\
\hline $\mathrm{Dim}$ & Experiment ${ }^{d}$ & $2.081(10)$ & 84.7 & $0.14(1)$ \\
\hline Dim & Experiment $^{e}$ & $2.072(7)$ & $96.0(3)$ & $0.15(1)$ \\
\hline
\end{tabular}

${ }^{2}$ Bond lengths in Ångstroms, bond angles in degrees, $M$ denotes the alkali atom, and $\mathrm{X}$ denotes the halide. See the text for definitions of the basis set.

${ }^{b}$ Microwave $R_{e}$ value quoted in Refs. 3(a) and 33.

'Electron diffraction corrected $R_{e}$ ( $l$ values fixed) from Ref. 11 .

${ }^{\mathrm{d}}$ Electron diffraction $R_{\alpha}(l$ values fixed) from Ref. 11.

eElectron diffraction $R_{e}$ from Ref. 14.

certain trends as a function of $s p$ basis size and augmentation with diffuse and polarization functions. Rather than present results for all species, an example is presented in Table I, using $\mathrm{NaF}$ and $\mathrm{Na}_{2} \mathrm{~F}_{2}$. Abbreviated results will be presented for the other dimers in what follows.

From the bond lengths in Table I, one can see that even the $3-21 \mathrm{G}$ basis gives results within $0.06 \AA$ of the spectroscopic value quoted by Huber and Herzberg ${ }^{33}$ for the monomer. In augmenting this basis, we first consider the addition of the standard diffuse functions $(3-21+G)$ (bond length expands) and addition of the standard polarization functions (3-21G*) (bond length decreases). Combining these functions $\left(3-21+G^{*}\right)$ leads to a bond length somewhat shorter than experiment, but the main point to note is the opposite effects of polarization and diffuse functions. One can understand the expansion upon addition of diffuse functions on a physical basis since $\mathrm{F}^{-}$should be larger than $F$, but if the functions in the basis are too tight, then $\mathrm{F}^{-}$in the ionic compound will shorten its bond length to make use of the basis functions on $\mathrm{Na}$. In a similar fashion, one expects a polarization of the orbitals upon bond formation, but without $d$ functions this cannot occur. Addition of $d$ functions allows the occupied orbitals to polarize and the bond shortens. The 3-21G "POL" result involves a calculation with two $d$ functions on $\mathrm{Na}$ (but no diffuse functions), and it is seen that the use of this basis leads to a somewhat larger bond length than the standard set. It will also be seen below that the POL basis functions have a significant effect on the dimer bond angle.

Turning to the 6-31G basis results, it is seen that this basis behaves similarly to the $3-21 \mathrm{G}$ basis, but overall leads to somewhat larger bond lengths which are in better agreement with experiment. Augmentation with diffuse functions on $F$ leads to a bond expansion, and use of the "polarized" two $d$ functions on $\mathrm{Na}$ (the standard and polarized functions are the same for $F$ ) leads to a further improvement in the bond length to a point where the equilibrium bond length differs from that in Huber and Herzberg by only $0.003 \AA$. This is an important effect to note, one which we found in all our calculationsinclusion of polarization functions appropriate for the $n-1$ shell of the alkali atom leads to a small, but important bond length change, and the POL basis in all cases includes functions that account for this effect.

The EXT basis set introduces no new results relative to the POL basis. Splitting the $F \boldsymbol{d}$ function into two functions (EXT/EXT2 results) also leads to no significant changes in bond length and about a $1^{\circ}$ angle change in the dimer. The similarity of these results for the two basis sets is encouraging since the EXT basis is essentially saturated in the $s$ and $p$ spaces for the atoms, and yet the much smaller basis yields geometries that are in quite good agreement with each other.

Examining the dimer results in Table I, one sees similar trends to those observed for the monomer upon addition of diffuse functions and polarization functions. Except for the $3-21+G$ basis, the expansion of the $M-X$ bond length upon dimer formation is a nearly uniform 0.14 $\pm 0.03 \AA$, independent of the initial bond length. Similar results are found for the other dimers as the basis sets are varied.

In comparing geometries, care must be taken to note the difference between directly comparable IR, microwave, and theoretically computed $R_{e}$ values and the thermally averaged $R_{a}$ value which is the primary distance parameter measured in electron diffraction. For the diatomic monomers, $R_{a}$ can be converted to $R_{e}$ in a straightforward manner (see Table I, of Ref. 12; note that thermally averaged bond lengths are expected to be longer than $R_{e}$ values in all cases). This is problematic for the polyatomic dimers, however, so in most cases we instead compare the change in bond length upon dimer formation [either $R_{e, \mathrm{MX}}$ (dimer) $-R_{e, \mathrm{MX}}$ (monomer) or $R_{a, \mathrm{MX}}$ (dimer) $-R_{a, \mathrm{MX}}$ (monomer)] below, since the effect of thermal averaging on the monomer and dimer bond lengths should roughly cancel in forming the $R_{a}$ difference. The one exception to this rule is when we compare with recent data on $\mathrm{Na}_{2} \mathrm{~F}_{2},{ }^{14}$ where an approximate correction was made to yield dimer $R_{e}$ values. In this case, we compare with the estimated experimental $R_{e}$ and $\Delta R_{e}$.

In comparison then with the experimental results for the dimer, one sees that the POL and EXT results are in 
TABLE II. Alkali halide monomer bond lengths and vibrational frequencies-RHF.

\begin{tabular}{lcccccc}
\hline \hline & $R_{e}{ }^{\mathrm{b}}$ & $\omega_{e}^{\mathrm{b}}$ & $R_{e}{ }^{\mathrm{b}}$ & $\omega_{e}^{\mathrm{c}}$ & $R_{e}{ }^{\mathrm{d}}$ & $\omega_{e}{ }^{\mathrm{d}}$ \\
\hline $\mathrm{LiF}$ & 1.565 & 937 & 1.564 & 910 & 1.554 & 938 \\
$\mathrm{LiCl}$ & 2.041 & 639 & 2.021 & 643 & 2.036 & 650 \\
$\mathrm{NaF}$ & 1.923 & 545 & 1.926 & 536 & 1.923 & 540 \\
$\mathrm{NaCl}$ & 2.400 & 353 & 2.361 & 366 & 2.389 & 358 \\
$\mathrm{KF}$ & 2.238 & 413 & 2.171 & 428 & 2.204 & 412 \\
$\mathrm{KCl}$ & 2.779 & 259 & 2.667 & 281 & 2.739 & 261 \\
\hline \hline
\end{tabular}

${ }^{2}$ Bond lengths are in Ångstroms, vibrational frequencies in $\mathrm{cm}^{-1}$.

${ }^{b}$ Present results-RHF in the POL basis set. The energies (in hartrees) at the optimized geometries are $\mathrm{LiF},-106.94974 ; \mathrm{LiCl},-467.01480$; $\mathrm{NaF},-261.32363 ; \mathrm{NaCl},-621.40342 ; \mathrm{KF},-698.62866 ; \mathrm{KCl}$, -1058.71070 .

'Experiment (Ref. 33).

${ }^{d}$ Langhoff et al. (Ref. 34) (RHF result).

quite good agreement for the bond length expansion. The $\mathrm{M}-\mathrm{X}-\mathrm{M}$ bond angle is larger than that reported in Ref. 11, but is in good agreement with that given in Ref. 14. The consistency of our results prompted a reanalysis of the experimental data from Ref. 11 and the new results are in much better agreement with the theory (see Discussion).

Based on the results obtained above and on similar test calculations not reported for other systems, we consider only the POL basis below. Test calculations in EXT quality basis sets have been performed, but are not reported here, as they produced similar results to the POL basis.

\section{B. Monomer and dimer results}

In Table II, we present RHF results in the "polarized" basis for the bond lengths and vibrational frequencies of $\mathrm{LiF}, \mathrm{LiCl}, \mathrm{NaF}, \mathrm{NaCl}, \mathrm{KF}$, and $\mathrm{KCl}$. Our results are compared with the most recent experimental values and also the theoretical results Langhoff et al. ${ }^{34}$ (RHF in large Slater basis sets). It is seen that in most cases, the bond lengths are reproduced quite well, the notable exception being $\mathrm{KCl}$, which is too long by $0.11 \AA$. The agreement with the larger Slater basis results also suggests that the present basis sets are adequate at the RHF level. Note that both theoretical studies yield excellent agreement for the vibrational frequencies of the monomers, even at the RHF level. The worst case is again $\mathrm{KCl}$, but the error is still less than $10 \%$.

As a qualitative correction for the larger species, one might note that $\mathrm{LiF}$ and $\mathrm{NaF}$ are calculated to within an accuracy of $0.01 \AA$. If one took this to indicate that these basis sets and the RHF treatment were sufficient for these species, then any larger errors for species containing other atoms could be associated with the insufficiency of the basis sets or use of RHF wave functions in those cases. Thus, the errors of $0.019 \AA$ in $\mathrm{LiCl}$ and $0.038 \AA$ in $\mathrm{NaCl}$ could be associated with an error in $\mathrm{Cl}$, and the error of $0.067 \AA$ in $\mathrm{KF}$ would be attributed to an error in $\mathrm{K}$, leading to a predicted error of $0.086-0.105 \AA$ for $\mathrm{KCl}$ compared to the actual error of $0.112 \AA$.

To further probe our difficulties in treating $\mathrm{K}$-containing species, we have performed calculations adding an $f$ function $(\alpha=0.375)$ on $\mathrm{K}$ in a basis similar to the
TABLE III. Dimer geometries and bond length expansions-RHF. ${ }^{a}$

\begin{tabular}{llrl}
\hline \hline & $R_{\mathrm{MX}}$ & $\Theta_{\mathrm{XMX}}$ & $\Delta R_{\mathrm{MX}}$ \\
\hline $\mathrm{Li}_{2} \mathrm{~F}_{2}$ & 1.717 & 99.5 & 0.152 \\
$\mathrm{Li}_{2} \mathrm{Cl}_{2}$ & 2.222 & 107.5 & 0.181 \\
$\mathrm{Na}_{2} \mathrm{~F}_{2}$ & 2.065 & 93.1 & 0.142 \\
$\mathrm{Na}_{2} \mathrm{Cl}_{2}$ & 2.574 & 100.8 & 0.174 \\
$\mathrm{~K}_{2} \mathrm{~F}_{2}$ & 2.416 & 85.6 & 0.178 \\
$\mathrm{~K}_{2} \mathrm{Cl}_{2}$ & 2.970 & 92.3 & 0.191 \\
\hline \hline
\end{tabular}

${ }^{2}$ Bond lengths in Ångstroms, bond angles in degrees, $M$ denotes the alkali atom, and $\mathrm{X}$ denotes the halide. Results are from RHF calculations in the POL basis set.

POL basis (having only a single $d$ function $\alpha=0.4$ instead of the two functions of the POL basis), but found no significant change in the geometry. In a basis for $\mathrm{K}$ analogous to the EXT basis sets for $\mathrm{Na}$ or $\mathrm{F}$, we have also split the contraction of the $\mathrm{K} 3 s$ orbital (the $3 p$ was already split), but found no appreciable change in geometry. It is possible that further core polarization is required, or that our basis is not sufficiently tight in the core region, leading to too large a radius for the cation, but we have been unable to significantly shorten the bond length below that presented in Table II.

In Table III, we present our results for the dimers of these species in the POL basis. We also include in this table the expansions of the $\mathrm{M}-\mathrm{X}$ bond lengths in going from monomers to dimers. It is seen that in general the bond lengths expand by from $0.16 \pm 0.03 \AA$. It will be seen below that we obtain good agreement with the experimental dimer bond length for $\mathrm{KCl}$, but this is due largely to the error we make in the monomer bond length. That is, the experimental expansion for $\mathrm{KCl}$ is predicted to be 0.247 $\pm 0.063 \AA$, which is in agreement with our RHF results at the low end of the uncertainty, but significantly above the MP2 value presented below.

Table IV contains vibrational frequencies for all dimers at the RHF level in the POL basis set. The irreducible representations appropriate to each $D_{2 h}$ symmetry mode are listed above the frequencies. It is not known what effect correlation has on the vibrational frequencies of the dimers, but one might expect the effect to be small given the simple bonding in the dimers and the small effect that correlation has on the monomer frequencies in the study by Langhoff et al. $^{34}$

TABLE IV. Dimer vibrational frequencies-RHF. ${ }^{\mathrm{a}}$

\begin{tabular}{lccrccc}
\hline \hline & $B_{3 u}$ & $B_{2 u}$ & $B_{1 u}$ & $A_{g}$ & $A_{g}$ & $B_{1 g}$ \\
\hline $\mathrm{Li}_{2} \mathrm{~F}_{2}$ & 698 & 600 & 305 & 691 & 382 & 586 \\
$\mathrm{Li}_{2} \mathrm{Cl}_{2}$ & 513 & 374 & 186 & 458 & 213 & 427 \\
$\mathrm{Na}_{2} \mathrm{~F}_{2}$ & 394 & 383 & 162 & 404 & 219 & 350 \\
$\mathrm{Na}_{2} \mathrm{Cl}_{2}$ & 274 & 228 & 96 & 258 & 134 & 230 \\
$\mathrm{~K}_{2} \mathrm{~F}_{2}$ & 278 & 312 & 114 & 300 & 145 & 261 \\
$\mathrm{~K}_{2} \mathrm{Cl}_{2}$ & 189 & 186 & 67 & 192 & 93 & 168 \\
\hline \hline
\end{tabular}

${ }^{2}$ Vibrational frequencies in $\mathrm{cm}^{-1}$. The molecules are oriented so that the $z$ axis is perpendicular to the molecular plane and the alkali atoms lie along the $y$ axis. Results are from RHF calculations in the POL basis set. 


\section{Correlation effects}

One might ask whether inclusion of correlation would significantly alter the geometries obtained above. To begin to answer this question, we have performed second-order Møller-Plesset (MP2) geometry optimizations in the POL basis. We have correlated the highest $s$ and $p$ shells of the halide atom and the $n-1 s$ and $p$ shells of the alkali atoms in all cases. These results are presented in Table $V$ for the monomer geometries, along with the singles and doubles configuration interaction (CISD) results of Langhoff et $a l .^{34}$ It is seen that the largest bond length change upon correlation occurs for $\mathrm{KCl}$ and yields a bond length decrease of similar size to that found in the study by Langhoff et $a l .{ }^{34}$ Inclusion of correlation does not account for all of our error relative to experiment, the remainder of which must lie in some combination of basis set incompleteness, lack of relativistic contributions, and a limited correlation treatment.

Results from MP2 calculations in the POL basis for the dimers are presented in Table VI, along with the bond length change relative to the MP2 monomer calculations. It is seen that the bond length changes are similar to those seen in the RHF results.

In Table VII, we present dimerization energies, uncorrected for basis set superposition effects, for all species considered here at the RHF and MP2 levels. We have also examined basis set superposition effects at the RHF level using a counterpoise correction, calculating the energies of the monomers at their equilibrium geometries with the full dimer basis present, the second monomer basis being placed at a distance of $R_{e}^{\text {dimer }}$ from the first, and then obtaining dimerization energies from these monomer energies. We find at most a $1 \mathrm{kcal} / \mathrm{mol}$ difference from the results of Table VII and thus present only the uncorrected results. In Table VII, it is seen that the RHF results are in quite good agreement with the MP2 results, except that those species involving $\mathrm{Cl}$ yield bond strengths about 6-8 $\mathrm{kcal} / \mathrm{mol}$ larger upon inclusion of correlation. The agreement with experiment is quite good. Similar agreement is found with other experimental dimerization energies quoted in Ref. 6.

TABLE V. Alkali halide monomer bond lengths--correlated results. ${ }^{a}$

\begin{tabular}{lcrcr}
\hline \hline & $R_{e}{ }^{\mathrm{b}}$ & \multicolumn{1}{c}{$\Delta R_{e}^{\mathrm{b}}$} & \multicolumn{1}{c}{$R_{e}{ }^{\mathrm{c}}$} & \multicolumn{1}{c}{$\Delta R_{e}{ }^{\mathrm{c}}$} \\
\hline $\mathrm{LiF}$ & 1.569 & 0.004 & 1.571 & 0.017 \\
$\mathrm{LiCl}$ & 2.023 & -0.018 & 2.033 & -0.003 \\
$\mathrm{NaF}$ & 1.942 & 0.019 & 1.921 & -0.002 \\
$\mathrm{NaCl}$ & 2.384 & -0.016 & 2.366 & -0.023 \\
$\mathrm{KF}$ & 2.250 & -0.012 & 2.184 & -0.020 \\
$\mathrm{KCl}$ & 2.739 & -0.040 & 2.697 & -0.042 \\
\hline \hline
\end{tabular}

${ }^{a}$ Bond lengths and bond length changes relative to the RHF results in the same basis in Ångstroms.

${ }^{b}$ Present results-MP2 in the POL basis set. The energies (in hartrees) at the optimized geometries are $\mathrm{LiF},-107.16379 ; \mathrm{LiCl},-467.16344$; $\mathrm{NaF},-261.56667 ; \mathrm{NaCl},-621.58251 ; \mathrm{KF},-698.94758 ; \mathrm{KCl}$, $-1058.96243$.

${ }^{\text {c}}$ Langhoff et al. (Ref. 34 ) CISD results.
TABLE VI. Dimer geometries and bond length expansions-MP2, ${ }^{\mathrm{a}}$

\begin{tabular}{llrl}
\hline \hline & $R_{\mathrm{MX}}$ & $\Theta_{\mathrm{XMX}}$ & $\Delta R_{\mathrm{MX}}$ \\
\hline $\mathrm{Li}_{2} \mathrm{~F}_{2}$ & 1.713 & 101.7 & 0.144 \\
$\mathrm{Li}_{2} \mathrm{Cl}_{2}$ & 2.185 & 108.0 & 0.162 \\
$\mathrm{Na}_{2} \mathrm{~F}_{2}$ & 2.094 & 92.9 & 0.152 \\
$\mathrm{Na}_{2} \mathrm{Cl}_{2}$ & 2.538 & 100.2 & 0.154 \\
$\mathrm{~K}_{2} \mathrm{~F}_{2}$ & 2.426 & 85.8 & 0.176 \\
$\mathrm{~K}_{2} \mathrm{Cl}_{2}$ & 2.907 & 91.9 & 0.168 \\
\hline \hline
\end{tabular}

${ }^{a}$ Bond lengths are in Ångstroms, bond angles in degrees, $\mathrm{M}$ denotes the alkali atom, and $X$ denotes the halide. Results are from RHF/MP2 calculations in the POL basis set.

\section{DISCUSSION}

In this section, we compare our results to experimental results on the dimers and to past theoretical results for the monomers and dimers.

\section{A. Comparison with experiment}

A variety of groups have used gas-phase electron diffraction to study the dimer geometries. The results of these studies are shown in Table VIII. Comparing these experimental results with our results, it is seen that:

(1) Our predicted RHF bond lengths and bond length expansions are in quite good agreement with experiment for $\mathrm{Li}_{2} \mathrm{~F}_{2}, \mathrm{Li}_{2} \mathrm{Cl}_{2}, \mathrm{Na}_{2} \mathrm{~F}_{2}$, and $\mathrm{Na}_{2} \mathrm{Cl}_{2}$. The experimental (thermally averaged) $R_{\mathrm{MX}}$ values are a little larger than the RHF $R_{\mathrm{MX}}$ lengths as expected. The bond length expansions all agree to $\pm 0.03 \AA$.

(2) Our predicted MP2 bond lengths and bond length expansions are in reasonable agreement with experiment for $\mathrm{Li}_{2} \mathrm{~F}_{2}, \mathrm{Li}_{2} \mathrm{Cl}_{2}, \mathrm{Na}_{2} \mathrm{~F}_{2}$, and $\mathrm{Na}_{2} \mathrm{Cl}_{2}$ except that the experimental $R_{a}$ is shorter than the computed $R_{e}$ for $\mathrm{Na}_{2} \mathrm{~F}_{2}$. The absolute lengths as well as the expansions are all good to $\pm 0.04 \AA$. With the exception of $\mathrm{Na}_{2} \mathrm{~F}_{2}$, correlation leads to a small decrease in the extent of bond length expansion upon dimer formation, but one would not draw any new

TABLE VII. Dimetrization energies.

\begin{tabular}{lccc}
\hline & Method & $\Delta E(\mathrm{kcal} / \mathrm{mol})$ & $\Delta E_{\text {expt }}$ (kcal/mol) \\
\hline $\mathrm{Li}_{2} \mathrm{~F}_{2}$ & $\mathrm{RHF}$ & 63.0 & 63.6 \\
$\mathrm{Li}_{2} \mathrm{~F}_{2}$ & $\mathrm{MP2}$ & 63.4 & \\
$\mathrm{Li}_{2} \mathrm{Cl}_{2}$ & $\mathrm{RHF}$ & 47.8 & 54.4 \\
$\mathrm{Li}_{2} \mathrm{Cl}_{2}$ & $\mathrm{MP2}$ & 54.6 & \\
$\mathrm{Na}_{2} \mathrm{~F}_{2}$ & $\mathrm{RHF}$ & 60.4 & 59.3 \\
$\mathrm{Na}_{2} \mathrm{~F}_{2}$ & $\mathrm{MP2}$ & 59.8 & \\
$\mathrm{Na}_{2} \mathrm{Cl}_{2}$ & $\mathrm{RHF}$ & 46.6 & 51.7 \\
$\mathrm{Na}_{2} \mathrm{Cl}_{2}$ & $\mathrm{MP2}$ & 52.4 & \\
$\mathrm{~K}_{2} \mathrm{~F}_{2}$ & $\mathrm{RHF}$ & 50.7 & 49.3 \\
$\mathrm{~K}_{2} \mathrm{~F}_{2}$ & $\mathrm{MP2}$ & 51.1 & \\
$\mathrm{~K}_{2} \mathrm{Cl}_{2}$ & $\mathrm{RHF}$ & 42.7 & 45.4 \\
$-\mathrm{K}_{2} \mathrm{Cl}_{2}$ & $\mathrm{MP2}$ & 46.0 & \\
\hline
\end{tabular}

${ }^{\mathrm{a} B o n d}$ lengths in Ångstroms bond angles in degrees, $\mathrm{M}$ denotes the alkali atom, and $X$ denotes the halide. Results are from RHF/MP2 calculations in the POL basis sets.

${ }^{b} \mathrm{Data}$ for all F-containing species are from Ref. 35. Data for $\mathrm{NaCl}$ are from Ref. 36. Data for all other Cl-containing species are from Ref. 37. The values reported are those corrected to $0 \mathrm{~K}$ (taken from Ref. 38). 
TABLE VIII. Experimental dimer geometries and bond length expansions. ${ }^{a}$

\begin{tabular}{lclccrr}
\hline \hline & Reference & \multicolumn{1}{c}{$R_{a, \mathrm{MX}}$} & $\Theta_{a, \mathrm{XMX}}$ & $\Delta R_{a, \mathrm{MX}}$ & Exp-RHF $^{\mathrm{b}}$ & Exp-MP2 $^{\mathrm{b}}$ \\
\hline $\mathrm{Li}_{2} \mathrm{~F}_{2}$ & 9 & $1.746(15)$ & $104.7(2.4)$ & 0.16 & 0.01 & 0.02 \\
$\mathrm{Li}_{2} \mathrm{Cl}_{2}$ & 10 & $2.23(3)$ & $108.0(4.0)$ & $0.18^{\mathrm{c}}$ & 0.00 & 0.02 \\
$\mathrm{Na}_{2} \mathrm{~F}_{2}$ & 11 & $2.081(10)$ & $84.6(6)$ & 0.14 & 0.00 & -0.01 \\
$\mathrm{Na}_{2} \mathrm{~F}_{2}$ & 14 & $2.072(7)$ & $96.0(3.0)$ & 0.14 & 0.01 & 0.00 \\
$\mathrm{Na}_{2} \mathrm{Cl}_{2}$ & 12 & $2.584(34)$ & $101.4(2.4)$ & 0.20 & 0.02 & 0.04 \\
$\mathrm{~K}_{2} \mathrm{~F}_{2}$ & 11 & $2.347(28)$ & $84.7(1.6)$ & 0.16 & 0.02 & 0.02 \\
$\mathrm{~K}_{2} \mathrm{Cl}_{2}$ & 12 & $2.950(54)$ & $96.0(2.4)$ & 0.25 & 0.06 & 0.08 \\
\hline \hline
\end{tabular}

Bond lengths in Ångstroms, bond angles in degrees.

${ }^{b}$ Differences between experimental and theoretical bond length expansions with the experimental expansions based on $R_{a}$ values and the theoretical expansions based on $R_{e}$ values. A positive value indicates a larger experimental expansion.

'The monomer $R_{a}$ value was calculated from the experimental $R_{e}$ value [Ref. 3(a)] and the $R_{a}-R_{e}$ value of $0.0275 \AA$ calculated using the expressions in Ref. 12 .

conclusions concerning the dimers based on the correlated results.

(3) In general, our bond angles support the experimental results with the exception of the results from Ref. 11 for $\mathrm{Na}_{2} \mathrm{~F}_{2}$. However, a reanalysis of the experimental data (discussed below) shows that the experimental data are unable to choose between an angle of approximately $85^{\circ}$ and one of approximately $95^{\circ}$. The results of Ref. 14 also support the larger angle. We therefore suggest that the approximately $95^{\circ}$ angle is the correct value.

(4) The absolute bond length errors are somewhat larger for $\mathrm{KF}$ and $\mathrm{K}_{2} \mathrm{~F}_{2}$. However, we obtain a bond length expansion similar to that seen experimentally, as well as good agreement for the bond angle, even though $R_{a}$ is shorter than $R_{e}$.

(5) $\mathrm{KCl}$ and $\mathrm{K}_{2} \mathrm{Cl}_{2}$ represent the most serious disagreements with experiment, although the experimental bond length error bar is fairly large due to the similar scattering powers of $\mathrm{K}$ and $\mathrm{Cl}$. While the absolute errors in bond lengths could be inferred from the results on the KF and $\mathrm{NaCl}$ monomers (see above), the bond length expansion differences in Table VIII are twice as large as for any other compound. Our predicted expansion at the RHF level is essentially within the $\pm 0.06 \AA$ error limits from the experimental analysis, but at the very lowest end of the uncertainty range, and our MP2 value is significantly outside the uncertainty range. One could argue that our large error in bond length mitigates against use of our results for the expansion. Indeed, we do find significant fluctuations in the bond length expansion, especially in smaller basis sets. However, in test calculations using large basis sets for $\mathrm{K}$ and $\mathrm{F}$ in $\mathrm{KF}$ and its dimer, we have found some sensitivity to the choice of $d$ functions used in the absolute bond lengths, but similar bond length expansions when using either diffuse or tight $d$ functions. We feel it is reasonable to assume that the predicted expansion is good to $\pm 0.03 \AA$ and therefore suggest that although the combined theoretical and experimental error bars just overlap, the current experimental value may well be too high.

The consistency of the $a b$ initio prediction of a $\Theta_{\mathrm{FNaF}}$ bond angle greater than $90^{\circ}$ for $\mathrm{Na}_{2} \mathrm{~F}_{2}$ led us to reanalyze the experimental data for this species. The problem here is not the simple confusion of the complementary $\mathrm{Na}-\mathrm{F}-\mathrm{Na}$ angle with the $\mathrm{F}-\mathrm{Na}-\mathrm{F}$ angle, but rather the similarity in this case of the scattering factors for $\mathrm{Na}$ and $\mathrm{F}$. Scattering for atoms goes roughly as the atomic number $Z$ squared, and $\mathrm{Na}$ and $\mathrm{F}$ are only separated by $\mathrm{Ne}$ in the Periodic Table. Electron diffraction data are analyzed by calculating the scattering from independent atoms (the independent atom model or IAM) and varying the geometrical parameters (distances and mean amplitudes of vibration) for these atoms until the experimental intensities are best reproduced. Particularly where it is not possible to resolve each individual distance (which is exacerbated for these hot, floppy molecules by the large mean amplitudes of vibration) and when scattering factors are very similar, it can happen that more than one valid fit can be found, and in this case, the $\mathrm{NaF}$ data are fit almost equally well by leaving all other parameters essentially unchanged and changing the $\mathrm{F}-\mathrm{Na}-\mathrm{F}$ angle from $84.6(6)^{\circ}$ to $94.7(7)^{\circ}$. This is equivalent to swapping the $r(\mathrm{Na}-\mathrm{Na})$ and $r(\mathrm{~F}-\mathrm{F})$ nonbonded distances, which makes little difference to the total intensities because of the very similar scattering factors. Without the $a b$ initio work presented here, it would only be possible to present both options, with a very slight edge to smaller angle. Combining the experimental and theoretical results, however, we feel reasonably confident in choosing the larger angle as the more accurate value. The new $94.7^{\circ}$ angle also better fits expected trends for the alkali fluoride dimer F-X-F angle (see Fig. 4 of Ref. 11).

The analogous case for the alkali chlorides is $\mathrm{KCl}$. From the experimental point of view, ${ }^{12}$ this was, as expected, the least tractable in the series to analyze, requiring all mean amplitudes of vibration to be fixed in order to obtain convergence. Once again, the monomer and dimer $R_{\mathrm{KCl}}$ distances are correlated, i.e., not resolved, and hence share one asymmetric peak in the experimental radial distribution curve. However, when corrected for thermal averaging, the electron diffraction value for the monomer bond distance agrees within $0.002 \AA$ with the very precise microwave value, leaving primarily the mean amplitudes of vibration to consider. It is quite straightforward to calculate the monomer mean amplitude of vibration, and for the dimers, the same program was used to calculate all of the alkali halide vibrational amplitudes, so there should not be a unique problem with $\mathrm{KCl}$. 
The possible effect of multiple scattering corrections are currently being considered for the heavier alkali halide dimers, but such effects are generally much smaller than the kind of change needed here (see, for instance, Miller and Fink $^{39}$ ) and should not be especially large for $\mathrm{KCl}$. The effect of using independent ions (the independent ion model or $\mathrm{IIM}^{40}$ ) instead of atoms to calculate the scattering is also being considered for these highly ionic molecules. For the $\mathrm{NaCl}$ dimer, Miki et al. ${ }^{41}$ found that using the IIM caused the bonded $\mathrm{Na}-\mathrm{Cl}$ distance to decrease by $0.023 \AA$ relative to the IAM value, although their use of the nonphysical ionic phase shifts for this neutral molecule (seen in the big increase in the amount of dimer) make this more of a qualitative value. Interestingly, the $\mathrm{KBr}$ and $\mathrm{KI}$ dimer structures are also somewhat far from the predictions, but a comparison of the differences between the independent ion and atom models does not show anything unusual or particularly large (even compared to covalent molecules) for the potassium halides. On the other hand, the correlation of parameters in the monomer-dimer mixtures makes all of these small scattering effects worth pursuing.

An additional correction is the shrinkage effect arising from the fact that on the average, these highly vibrationally excited planar molecules are folded out of the plane, which tends to effectively shorten the nonbonded "crossdiagonal" distances in particular. Hargittai ${ }^{42}$ has found for the $\mathrm{NaCl}$ dimer using the experimental temperature of Miki et al. ${ }^{41}$ that the $\mathrm{Na}-\mathrm{Cl}$ bonded distance is effectively lengthened $0.015 \AA$ by this effect when calculated using harmonic potentials. Work is also underway to calculate this shrinkage effect using anharmonic $a b$ initio potentials, although preliminary results indicate only minor differences between the harmonic and anharmonic cases. Note that this effectively cancels the IIM shift, which is good in the sense that theory and experiment already agree quite nicely for the $\mathrm{NaCl}$ dimer. It does, however, bear on the likelihood that a number of probably small effects which may well cancel, but could also bring the current theoretical and experimental values for the $\mathrm{KCl}$ dimer into agreement. More definite statements must await further research on these various effects.

Comparison with experimental vibrational frequencies for the dimers is somewhat difficult as there are limited values for these species experimentally ${ }^{17-21}$ and there is some disagreement between values where duplicate measurements have been made. ${ }^{18,19}$ Compared with Snelson's ${ }^{19}$ matrix isolation spectra, we obtain good agreement for the infrared active modes (estimated gas-phase values $B_{3 u}$ $=655 \mathrm{~cm}^{-1}, B_{2 u}=570 \mathrm{~cm}^{-1}$, and $B_{1 u}=300 \mathrm{~cm}^{-1}$ ), but have larger differences between our results and the estimates he provided for the inactive modes. Similar conclusions apply to the work of Livensky. ${ }^{20}$ Cyvin et al. ${ }^{21}$ have also examined $\mathrm{Na}_{2} \mathrm{~F}_{2}$ and we again obtain good agreement with the observed frequencies $\left(B_{3 u}=380 \mathrm{~cm}^{-1}, B_{2 u}=363\right.$ $\mathrm{cm}^{-1}$, and $B_{1 u}<190 \mathrm{~cm}^{-1}$ ), but larger differences between our results and their estimates of the inactive modes. Based on these (limited) comparisons, it appears that our frequencies are more accurate than the semiempirical theories discussed below, but it will be helpful to study mixed dimers experimentally and theoretically where all the modes are IR active, and where one can begin to address these questions over a wider range of compounds.

Finally we note that the theoretical estimates of the dimerization energies are quite good. For the F-containing species, either RHF or MP2 results yield uniformly excellent agreement with the available experimental values, while for Cl-containing species, the MP2 values are somewhat better. In any event, these results suggest that these levels of theory could be used to study binding energies in other clusters and be expected to yield reasonable estimates of heats of aggregation there as well, as is also indicated by the results of Ref. 24 .

\section{B. Comparison with theory}

A few $a b$ initio studies have appeared on the neutral alkali halide dimers ${ }^{22-24}$ (some groups have also studied dimer cations and anions ${ }^{23,43}$ ). One study was performed by Swepston $e t a l^{22(a)}$ based on Hartree-Fock wave functions using small basis sets with no polarization functions to study the geometry and binding energy of $\mathrm{Li}_{2} \mathrm{~F}_{2}$ and $\mathrm{Li}_{3} \mathrm{~F}_{3}$. They obtained quite good values of $R_{\mathrm{MX}}$ for the monomer and dimer, but somewhat underestimated the bond angle and overestimated the energy of dimerization by approximately $15 \mathrm{kcal} / \mathrm{mol}$. Sunil and Jordan ${ }^{23}$ examined $\mathrm{Na}_{2} \mathrm{Cl}_{2}$ in the process of studying $\mathrm{Na}_{2} \mathrm{Cl}_{2}^{+}$and $\mathrm{Na}_{2} \mathrm{Cl}_{2}^{-}$. In addition, Ochsenfeld and Ahlrichs ${ }^{24(\mathrm{a})}$ and Weis et al. ${ }^{24(\mathrm{~b})}$ have recently presented results for $\mathrm{Na}_{2} \mathrm{Cl}_{2}$ in the process of studying a variety of $\mathrm{Na}_{m} \mathrm{Cl}_{n}$ clusters. Similar basis sets to those employed here were used and RHF, ${ }^{23,24}$ MP2, ${ }^{23,24}$ and coupled-pair functional theory $(\mathrm{CPF})^{24}$ were used to examine the ground state structures. Their results support those obtained here and suggest that the geometries are insensitive to the fine details of the basis sets used.

Many semiempirical models have been developed to treat alkali halide clusters. These include the Rittner, 6 $T$-Rittner, ${ }^{3}$ Rothberg, ${ }^{3}$ modified Rothberg, ${ }^{3}$ and the shell model of Welch et al. ${ }^{4}$ to name some of the more studied models. Rather than compiling data from all of these methods, we here summarize our conclusions concerning comparisons of our results to these methods. Using the experimental results of Table VIII as a standard (neglecting the results for $\mathrm{K}_{2} \mathrm{Cl}_{2}$ ), we find

(1) The modified Rothberg model of Brumer and Karplus $^{3}$ yields the most consistent agreement with our results and with experiment for the bond length expansion upon dimerization. Its bond angles tend to differ somewhat more from experiment than ours, and for the $K$-containing species, it seems to significantly underestimate the bond angles. Its absolute values of dimer bond lengths are as good as our own for the lighter atom systems and appear to be more accurate for the heavy atom systems.

(2) The shell model of Welch et al. ${ }^{4}$ yields reasonable results for the bond angles, but seriously overestimates the bond length expansion upon dimerization for all $\mathrm{F}$ containing species. It seems to be in better agreement for $\mathrm{Cl}$ containing species. The absolute bond lengths show significantly greater scatter than our own. 
(3) The Rittner model as applied most recently by Chauhan et al. ${ }^{6}$ seriously underestimates the bond length expansions in all $\mathrm{F}$-containing species and overestimates the expansions in all $\mathrm{Cl}$-containing species studied here. The bond angles are in reasonable agreement with experiment. In contrast to these results, the $T$-Rittner method of Brumer and $\mathrm{Karplus}^{3}$ yields expansions that appear somewhat large, but are more consistent than the results of Chauhan et al. ${ }^{6}$ for this parameter. On the other hand, the $T$-Rittner model leads to bond angles that are consistently $5^{\circ}$ to $10^{\circ}$ too large.

(4) Several of the semiempirical models predict bond length expansions for $\mathrm{K}_{2} \mathrm{Cl}_{2}$ that are in much better agreement with experiment than are our values. However, these methods tend to either generally overestimate bond length expansions or overestimate the Cl-containing dimer expansions in particular. Thus, while these results may appear to support the experimental value in this case, we believe the agreement to be an artifact.

It would appear from the above results that while these methods contain the essential elements needed to describe the dimers (and, one hopes, larger clusters), they all suffer from some defect in the potentials for at least one of the atoms. It may be possible using the experimental and $a b$ initio results to correct these potentials and produce accurate semiempirical theories which will be of much wider applicability than the $a b$ initio methods, especially for larger clusters.

Overall, the above results suggest that Hartree-Fock wave functions are capable of yielding accurate geometric information for these alkali halide dimers, especially for the Li- and Na-containing molecules. Inclusion of correlation was not a significant factor in predicting the dimer geometries, but it had some effect on the dimer dissociation energies. For the prediction of geometries, it is encouraging that one might be able to use RHF results for larger clusters of the lower atomic number alkali halides. That one need not include correlation is not only advantageous from a computational viewpoint, but suggests that the bonding is simple conceptually and arises from an essentially ionic picture. The requirement that we augment the basis sets with polarization functions (core type for alkali systems) also suggests that the atomic constituents must polarize somewhat in forming the molecule. Finally, the need to include diffuse functions on the halide atoms points up the (obvious) charge expansion of the halide when the bond is formed.

From the point of view of a minimum basis set required to obtain converged results, we have found that the augmented 3-21G basis is inadequate due to inaccuracies in the absolute bond lengths and the bond length expansions upon dimer formation. It appears, however, that 6-31G quality basis sets augmented with diffuse and polarization functions are sufficient to yield results in agreement with much larger basis sets.

\section{CONCLUSIONS}

Results for ground state geometries, vibrational frequencies, and dimerization energies of a series of symmet- rical alkali halide dimers have been presented based on $a b$ initio Hartree-Fock and MP2 wave functions. It was found that Hartree-Fock theory in a modest basis set with diffuse and polarization functions yields results comparable to much larger basis sets and that the theoretical results are in excellent agreement with the experimental results for the $\mathrm{Li}$ and $\mathrm{Na}$ dimers. Our best results for the K-containing species tend to have bond lengths that are too long for the monomers, and this effect is carried over for the dimers. We also find a nearly uniform expansion of the $\mathrm{M}-\mathrm{X}$ bond length in proceeding from monomer to dimer of $0.16 \pm 0.03$ $\AA$, independent of the alkali or halide involved. The calculated dimer dissociation energies are in excellent agreement with experiment. Inclusion of correlation appears to have a minimal effect on the computed geometries and a modest effect on the binding energies. The vibrational frequencies for the monomers are in excellent agreement with experimental gas-phase results and are in reasonable agreement with the avaliable frequencies for the dimers. A reanalysis of the electron diffraction data from Ref. 11 for $\mathrm{Na}_{2} \mathrm{~F}_{2}$ in light of the current $a b$ initio results leads to a significant change in the experimental value of the bond angle.

\section{ACKNOWLEDGMENTS}

R.J.C. would like to acknowledge the Donors of the Petroleum Research Fund, administered by the ACS for partial support of this work, as well as the National Science Foundation for partial support, Grant No. CHE-9011770. R.J.M. would like to acknowledge that this work was supported by a grant from the Research Corporation. A grant from the PEW Foundation to R.J.M. and R.J.C. was also used in partial support of this work. The calculations were performed on the Harvey Mudd Computational Chemistry Facility, the establishment of which was made possible by a grant from the National Science Foundation, Grant No. USE-8950661.

${ }^{1}$ (a) R. G. Gordon and Y. S. Kim, J. Chem. Phys. 56, 3122 (1972); (b) S. Trugman and R. G. Gordon, ibid. 64, 4625 (1976).

${ }^{2}$ J. Berkowitz, J. Chem. Phys. 29, 1386 (1958); 32, 1519 (1960).

${ }^{3}$ (a) P. Brumer and M. Karplus, J. Chem. Phys. 58, 3903 (1973); (b) 64, 5165 (1976).

${ }^{4}$ D. O. Welch, O. W. Lazareth, G. J. Dienes, and R. D. Hatcher, J. Chem. Phys. 64, 835 (1976).

${ }^{5}$ A. K. M. Islam, Can. J. Phys. 60, 865 (1982).

${ }^{6}$ R. S. Chauhan, S. C. Sharma, S. B. Sharma, and B. S. Sharma, J. Chem. Phys. 95, 4397 (1991).

${ }^{7}$ M. Kumar and J. Shanker, J. Chem. Phys. 96, 5289 (1992).

${ }^{8}$ J. M. Mestdagh and J. P. Visticot, Chem. Phys. 155, 79 (1991).

${ }^{9}$ V. G. Solomonik, K. S. Krasnov, G. Girichev, and E. Zasorin, J. Struct. Chem. 20, 361 (1979).

${ }^{10}$ S. H. Bauer, T. Ino, and R. F. Porter, J. Chem. Phys. 33, 685 (1960).

${ }^{11}$ J. G. Hartley and M. Fink, J. Chem. Phys. 89, 6058 (1988).

${ }^{12}$ R. J. Mawhorter, M. Fink, and J. G. Hartley, J. Chem. Phys. 83, 4418 (1985).

${ }^{13}$ (a) J. G. Hartley and M. Fink, J. Chem. Phys. 87, 5477 (1987); (b) 89, 6053 (1988).

${ }^{14}$ S. A. Lapshina, G. V. Girichev, and S. A. Shlykov, Zh. Strukt. Kim. 30, 397 (1989).

${ }^{15}$ N. G. Phillips, C. W. S. Conover, and L. A. Bloomfield, J. Chem. Phys. 94, 4980 (1991).

${ }^{16}$ J. P. Rose and R. S. Berry, J. Chem. Phys. 96, 517 (1992).

${ }^{17}$ S. Schlick and O. Schnepp, J. Chem. Phys. 41, 463 (1964).

${ }^{18}$ W. Klemperer and W. G. Norris, J. Chem. Phys. 34, 1071 (1961). 
${ }^{19}$ A. Snelson, J. Chem. Phys. 46, 3652 (1967)

${ }^{20}$ M. Linevsky, J. Chem. Phys. 38, 658 (1963).

${ }^{21}$ S. J. Cyvin, B. N. Cyvin, and A. Snelson, J. Phys. Chem. 74, 4338 (1970).

22 (a) P. N. Swepston, H. L. Sellers, and L. Schäfer J. Chem. Phys. 74, 2372 (1981); (b) C. P. Baskin, C. F. Bender, and P. A. Kollman, J. Am. Chem. Sóc. 95, 5868 (1973); (c) M. Rupp and R. Ahlrichs, Theor. Chim. Acta 46, 117 (1977).

${ }^{23}$ K. K. Sunil and K. D. Jordan, J. Phys. Chem. 91, 1710 (1987); Chem. Phys. 143, 366 (1988); 164, 509 (1989).

${ }^{24}$ (a) C. Ochsenfeld and R. Ahlrichs, J. Chem. Phys. 97, 3487 (1992); (b) P. Weis, C. Ochsenfeld, R. Ahlrichs, and M. Kappes, ibid. 97, 2553 (1992).

${ }^{25}$ L. Pauling, The Nature of the Chemical Bond (Cornell University, Ithaca, NY 1960).

${ }^{26}$ GAMESS is a general purpose electronic structure program. The original program was assembled by $\mathbf{M}$. Dupuis, D. Spangler, and J. J. Wendoloski at the National Resource for Computations in Chemistry, Software Catalog, University of California, Berkeley, CA, 1980, Program QGO1. The current version is described in the Quantum Chemistry Program Exchange Newsletter: M. W. Schmidt, K. K. Baldridge, J. A. Boatz, J. H. Jensen, S. Koseki, M. S. Gordon, K. A. Nguyen, T. L. Windus, and S. T. Elbert, QCPE Bull, 10, 52 (1990).

${ }^{27}$ The MELDF-X series of electronic structure codes was originally written by L. E. McMurchie, S. T. Elbert, S. R. Langhoff, and E. R. Davidson. It has been modified substantially by $D$. Feller, R. J. Cave, D. Rawlings, R. Frey, R. Daasch, L Nitzche, P. Phillips, K. Iberle, C. Jackels, and E. R. Davidson.

${ }^{28}$ (a) W. J. Hehre, R. Ditchfield, and J. A. Pople. J. Chem. Phys. 56, 2257 (1972); (b) P. C. Hariharan and J. A. Pople, Theor. Chim. Acta 28, 213 (1973).
${ }^{29}$ (a) J. S. Binkley, J. A. Pople, and W. J. Hehre, J. Am. Chem. Soc. 102, 939 (1980); (b) M. S. Gordon, J. S. Binkley, J. A. Pople, W. J. Pietro, and W. J. Hehre, ibid. 104, 2797 (1982); (c) K. D. Dobbs and W. J. Hehre, J. Comput. Chem. 7, 359 (1986); (d) P. C. Hariharan and J. A. Pople, Theor. Chim. Acta 28, 213 (1973); (e) T. Clark, J. Chandresekhar, G. W. Spitznagel, and P. v. R. Schleyer, J. Comput. Chem. 4, 294 (1983); (f) M. J. Frisch, J. A. Pople, and J. S. Binkley, J. Chem. Phys. 80, 3265 (1984)

${ }^{30}$ M. W. Schmidt and K. Reudenberg, J. Chem. Phys. 71, 3951 (1979).

${ }^{31}$ R. C. Raffenetti, J. Chem. Phys. 58, 4452 (1973).

${ }^{32}$ A. D. McLean and G. S. Chandler, J. Chem. Phys. 72, 5639 (1980).

${ }^{33}$ K. P. Huber and G. Herzberg, Molecular Spectra and Molecular Structure IV. Constants of Diatomic Molecules (Van Nostrand-Reinhold, New York, 1979).

${ }^{34}$ S. R. Langhoff, C. W. Bauschlicher, Jr., and H. Partridge, J. Chem. Phys. 84, 1687 (1986).

${ }^{35}$ M. Eisenstadt, G. M. Rothberg, and P. Kush, J. Chem. Phys. 29, 797 (1958).

${ }^{36}$ M. Eisenstadt, V. S. Rao, and G. M. Rothberg, J. Chem. Phys. 30, 605 (1959).

${ }^{37}$ S. H. Bauer, R. M. Diner, and R. F. Porter, J. Chem. Phys. 29, 991 (1958).

${ }^{38}$ G. M. Rothberg, J. Chem. Phys. 34, 2069 (1961).

${ }^{39}$ B. R. Miller and M. Fink, J. Chem. Phys. 57, 5326 (1981).

${ }^{40}$ A. Chesnyi and N. Rambidi, Chem. Phys. 26, 155 (1977).

${ }^{41}$ H. Miki, K. Kakumoto, T. Ino, S. Kodera, and J. Kakinoki, Acta Crystallogr. Sect. A 36, 96 (1980).

${ }^{42}$ M. Hargittai, Stereochemical Applications of Gas-Phase Electron Diffraction (VCH, New York, 1988), part B, p. $395 \mathrm{ff}$.

${ }^{43}$ G. Galli, W. Andreoni, and M. P. Tosi, Phys. Rev. A 34, 3580 (1986). 